\title{
Direct Atomic-Scale Observation of Droplets Coalescence Driven Nucleation and Growth of Supported Bismuth Nanocrystal in the TEM
}

\author{
Junjie $\mathrm{Li}^{1}$ and Francis Leonard Deepak ${ }^{1}$
}

1. Nanostructured Materials Group, Department of Advanced Electron Microscopy, Imaging and Spectroscopy, International Iberian Nanotechnology Laboratory (INL), Braga, Portugal.

Dynamic processes at the liquid/solid interfaces are of key significance across broad areas of technological interest, such as solidification, liquid-phase epitaxial growth, wetting, liquid-phase joining, crystal growth, and lubrication [1,2]. Many studies have been reported with the indirect evidence of density fluctuations at liquid/solid interfaces on the basis of X-ray scattering methods [3], atomic force microscopy (AFM) [4] and with the support of atomistic simulations [5]. Transmission electron microscopy (TEM) can, in principle, allow us to observe dynamic processes directly, yet to date such investigations are scarce due to the exceptionally high need of an elegant microscope and a suitable system that enables to see liquids, solids and their junctions simultaneously. Hence, due to the lack of direct experimental observations at the atomic-scale and also for the intricacies of tackling such challenging systems, much confusion still exists regarding the atomistic understanding of the dynamic processes at the liquid/solid interfaces.

Bismuth (Bi) bulk metal has a low melting point of $544.4 \mathrm{~K}$ and the melting temperature of its nanoparticles can be low even to room temperature due to size effects. As such, $\mathrm{Bi}$ is an ideal model material to track electron-beam induced nucleation and growth. Hence we take this unique system comprising of the formed $\mathrm{Bi}$ droplets on crystalline $\mathrm{SrBi}_{2} \mathrm{Ta}_{2} \mathrm{O}_{9}$ support and by manipulating electron doses within the TEM we probe such a liquid/solid interface.

In this work, we observe the in-situ atomic-scale behavior of fabricated Bi droplets segregated on $\mathrm{SrBi}_{2} \mathrm{Ta}_{2} \mathrm{O}_{9}$ by using aberration corrected transmission electron microscopy. We demonstrate ordered interface and surface structures for the droplets on the oxide at the atomic-scale and unravel a nucleation mechanism involving droplet coalescence at the liquid/solid interface (Figure 1). We identify a critical diameter of the formed nanocrystal in stabilizing the crystalline phase and reveal lattice induced fast crystallization of the droplet at the initial stage of the coalescence of nanocrystal with droplet. Further sequential observations show the stepped coalescence and growth mechanism of the nanocrystals at the atomic-scale (Figure 2). Interestingly, in contrast to the rapid coalescence of two liquid droplets, the coalescence of a nanocrystal with a liquid droplet takes place via a clear step-migration mechanism. These results offer insights into the dynamic process at liquid/solid interfaces, which may have implications for many functionalities of materials and their applications [6].

The capability of performing such in-situ atomic-scale observations of dynamic processes of liquid droplets at a liquid/solid interface using advanced transmission electron microscopy represents a significant step forward in understanding liquids, solids and their interactions at the atomic-scale. These findings provide detailed dynamic information at the $\mathrm{Bi} / \mathrm{SrBi}_{2} \mathrm{Ta}_{2} \mathrm{O}_{9}$ liquid/solid interface at atomicscale, and should help to advance our general understanding of dynamic process in other liquid/solid interfaces.

References: 
[1] SH Oh et al, Science 310 (2005) p. 661.

[2] OG Shpyrko et al, Science 313 (2006) p. 77.

[3] H Reichert et al, Nature 408 (2000) p. 839.

[4] S Biggs and P Mulvaney, J. Chem. Phys. 100 (1994) p. 8501.

[5] GC Sosso et al, Chem. Rev. 116 (2016) p. 7078.

[6] J Li, Z Wang, FL Deepak, ACS Nano 11 (2017) p.5590.

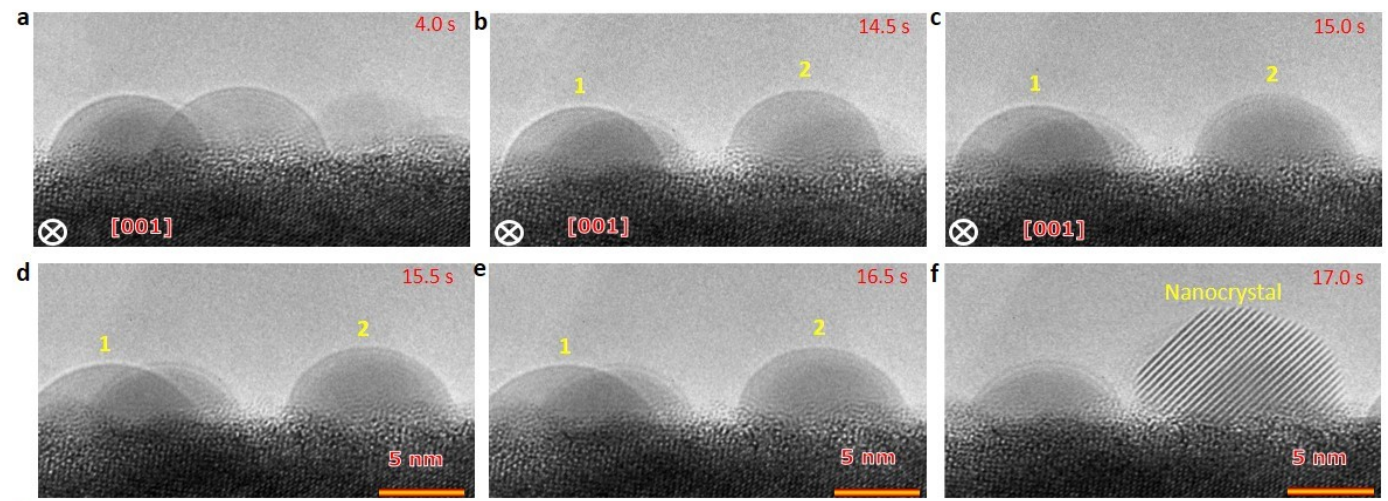

Figure 1. Coalescence induced crystallization (Droplet 1 and 2) at the $\mathrm{Bi} / \mathrm{SrBi}_{2} \mathrm{Ta}_{2} \mathrm{O}_{9}$ interface. The electron dose rate is $4.22 \times 10^{4} \mathrm{e}^{-} / \AA^{2} \cdot \mathrm{s}$.

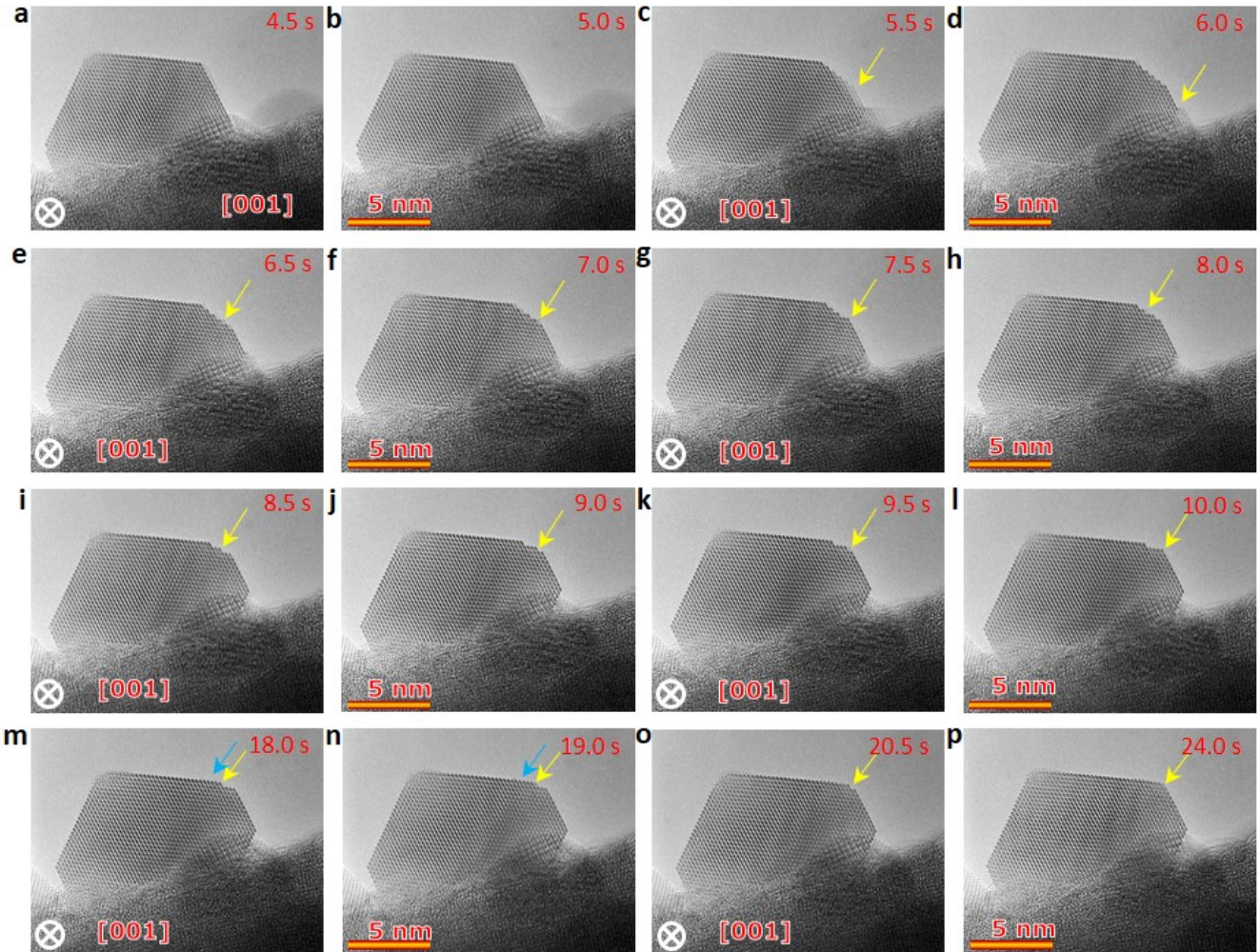

Figure 2. Coalescence of nanocrystal with droplet on the support and step-growth mechanism. a-e, HRTEM images showing the coalescence of nanocrystal with droplet on the support at the atomic scale. $\mathrm{f}-\mathrm{p}$, HRTEM images showing the atomic-scale observation of the step-growth of the supported $\mathrm{Bi}$ nanocrystal. The electron dose rate is $2.15 \times 10^{4} \mathrm{e}^{-} / \AA^{2} \cdot \mathrm{s}$. 